\title{
DNA Primase Small Subunit
}

National Cancer Institute

\section{Source}

National Cancer Institute. DNA Primase Small Subunit. NCI Thesaurus. Code C26562.

DNA primase small subunit (420aa, $\sim 50 \mathrm{kDa}$ ) is encoded by the human PRIM1 gene. This protein is involved in the biosynthesis of RNA primers for the Okazaki fragments made during DNA replication of the lagging strand. 